\title{
BMJ Open Impact of early palliative care on overall survival of patients with metastatic upper gastrointestinal cancers treated with first-line chemotherapy: a randomised phase III trial
}

\author{
Emilie Hutt, ${ }^{1}$ Arlette Da Silva, ${ }^{2}$ Emilie Bogart, ${ }^{3}$ Sara Le Lay-Diomande, ${ }^{4}$ \\ Diane Pannier, ${ }^{1}$ Stéphanie Delaine-Clisant, ${ }^{4}$ Marie-Cécile Le Deley, ${ }^{3,5}$ \\ Antoine Adenis ${ }^{1,6}$
}

To cite: Hutt E, Da Silva A, Bogart $\mathrm{E}$, et al. Impact of early palliative care on overall survival of patients with metastatic upper gastrointestinal cancers treated with first-line chemotherapy: a randomised phase III trial. BMJ Open 2018;8:e015904. doi:10.1136/ bmjopen-2017-015904

- Prepublication history for this paper is available online. To view these files please visit the journal online (http://dx.doi. org/10.1136/bmjopen-2017015904).

Received 19 January 2017

Revised 21 June 2017

Accepted 27 June 2017

Check for updates

${ }^{1}$ Department of Gastrointestinal Oncology, Centre 0scar Lambret Lille, France

${ }^{2}$ Palliative Care Unit, Centre Oscar Lambret, Lille, France ${ }^{3}$ Methodology and Biostatistic Unit, Centre Oscar Lambret,

Lille, France

${ }^{4}$ Clinical Research Unit, Centre Oscar Lambret, Lille, France ${ }^{5}$ CESP, INSERM, Faculté de médecine, Université Paris-Sud, Université Paris-Saclay, Villejuif, France

${ }^{6}$ Catholic University, Lille, France

Correspondence to

Professor Antoine Adenis;

scientifique@0-lambret.fr

\section{ABSTRACT}

Introduction Palliative care $(\mathrm{PC})$ has usually been offered at the end-of-life stage, although the WHO recommends providing $\mathrm{PC}$ as early as possible in the course of the disease. A recent study has shown that early PC (EPC) provides a more meaningful effect on quality of life and, surprisingly, on overall survival (OS) than standard treatment for patients with metastatic lung cancer. Whether EPC benefits also apply to patients with metastatic upper gastrointestinal (Gl) cancers is unknown. Methods and analysis EPIC is a randomised phase III trial comparing EPC plus standard oncologic care versus standard oncologic care in patients with metastatic upper Gl cancers. Its primary objective is to evaluate the efficacy of EPC in terms of OS. Its secondary objectives are to assess the effects of EPC on patient-reported outcomes (quality of life, depression and anxiety) and the effect of EPC on the number of patients receiving chemotherapy in their last 30 days of life. Assuming an exponential distribution of survival time, 381 deaths are required to ensure an $80 \%$ power for an absolute difference of $10 \%$ in 1 year $0 \mathrm{~S}$ rates $(40 \%$ vs $50.3 \%, \mathrm{HR}=0.75$; log rank test two-sided alpha $=5 \%$ ), leading to a planned sample size of 480 patients enrolled over 3 years and a final analysis at 4 years. The main analysis will be performed on the intentto-treat dataset.

Ethics and dissemination This study was approved by the 'Comité de Protection des Personnes Nord-Ouest I' (4 April 2016), complies with the Helsinki declaration and French laws and regulations and follows the International Conference on Harmonisation E6 (R1) Guideline for Good Clinical Practice. The trial results, even if they are inconclusive, will be presented at international oncology congresses and published in peer-reviewed journals.

Trial registration numbers EudraCT: 2015-A01943-46; Pre-results. NCT02853474.

\section{INTRODUCTION}

Medical care in the metastatic setting

Medical oncology aims to increase the survival rates of patients, even at metastatic

\section{Strengths and limitations of this study}

- Multicentric, nationwide, academic trial with a randomised design.

- Overall survival as a primary outcome, as it is a reliable and precise endpoint which has never been previously challenged in such setting.

- Providing an extra survival benefit with early palliative care (PC) would be a considerable contribution for patients, as would the implementation of these practices within the continuum of oncological care.

- Possible difficulties in recruiting participants due to the reluctance of some oncologists to talk about $\mathrm{PC}$ at diagnosis and possible screen failures due to patient refusals. Actions are ongoing to communicate on this issue and overcome this hurdle.

- Compared with Temel's pivotal study, the control arm in our study may include some components of $\mathrm{PC}$ visits as this is a clinical practice in France. This may lead to a smaller relative difference between randomised groups compared with the Temel's publication. The sample size calculation has been performed targeting a HR of death of 0.75 compared with an observed HR of 0.6 in Temel's study. The study will be underpowered for a smaller effect.

stages, in addition to reducing disease-related and treatment-related symptoms. However, providing palliative care (PC), which includes symptom management, nutritional support, psychosocial support and assistance with end-of-life preferences to improve quality of life, may be as important as survival issues at metastatic stages. Decades ago, PC services were initiated in France to provide a medical alternative to questionable medical practices regarding the end-of-life period: abandonment, euthanasia and inappropriate aggressive therapy. According to the French Society of Palliative Care, ${ }^{1}$ PC is a holistic approach 
that aims to provide active care to a person with a serious, progressive or terminal illness. The objective of PC is to relieve pain and other distressing symptoms; moreover, PC also accounts for psychological, social and spiritual suffering. PC offers an interdisciplinary support system to help patients and their relatives. ${ }^{1}$ In both France and in $\mathrm{USA}^{2}{ }^{2} \mathrm{PC}$ is usually offered late, at the end-of-life stage, although the WHO recommends providing PC as early as possible in the course of the disease to increase quality of life. ${ }^{3}$ In 1999, PC access became a right guaranteed by the law for patients and their families in France. ${ }^{4}$ This context explains why even now, PC often means 'end-oflife' for the patient and for caregivers and many doctors. The last WHO recommendations are less restrictive than the outdated 1996 French recommendations that stated that PC should be offered as early as possible in the course of the disease to increase quality of life and to positively influence the course of the illness. ${ }^{3}$ The WHO recommendations add that $\mathrm{PC}$ is applicable early in the course of illness in conjunction with other therapies that are intended to prolong life, such as chemotherapy $(\mathrm{Ct})$ or radiation therapy; the recommendations also state that investigations are necessary to better understand and manage distressing clinical complications. ${ }^{3}$

\section{THE CONCEPT OF EARLY PALLIATIVE CARE}

In a recent randomised study, 151 patients newly diagnosed with metastatic non-small-cell lung cancer were assigned to receive either early PC (EPC) integrated with standard oncologic care or standard oncologic care alone. ${ }^{5}$ It was hypothesised that patients who received EPC would have a better quality of life (primary endpoint) compared with patients who received standard oncologic care only. In the EPC group, the first visit with the PC services (board-certified PC physicians and advanced practice nurses) was planned within 3 weeks after enrolment and at least monthly thereafter; all but one patient had the first visit by the 12th week, with a mean of four total visits. In this study, the authors referred to the PC package presented in the recommendations from the National Consensus Project for Quality Palliative Care. ${ }^{6}$ For patients with metastatic non-small-cell lung cancer, EPC led to significant improvements in quality of life and in mood. Additionally, EPC led to a significantly longer survival (median survival, 11.6 vs 8.9 months; $\mathrm{HR}=0.60$, $\mathrm{p}=0.02$ ), despite less aggressive end-of-life care. ${ }^{5}$ Several hypotheses for the effect of EPC on survival have been raised by Pirl et al, ${ }^{7}$ such as improving the management of medical comorbidities including depression and aiding in the discontinuation of inappropriate and possibly detrimental cancer treatments at the end of life.

Following the publication of Temel and colleagues, ${ }^{5}$ the American Society of Clinical Oncology recommended that 'combined standard oncology care and PC should be considered earlier in the course of the illness for any patient with metastatic cancer.... ${ }^{8}$ However, it appears that a gap exists between these recommendations and current practice in France and elsewhere. Moreover, there is no consensus on how EPC should be integrated into oncologic services; a randomised trial recently reported a non-significant increase in survival rate for early (30-60 days after diagnosis) versus delayed (3 months later) initiation of $\mathrm{PC}$ in 207 patients diagnosed with various types of advanced cancer. ${ }^{2}$ The results of Temel's study have modified the perception of many oncologists about the objectives of PC. However, additional clinical studies seem necessary before considering EPC as an additional survival input in advanced malignancies other than metastatic non-small-cell lung cancers.

\section{METASTATIC UPPER GASTROINTESTINAL CANCERS}

The median survival time of patients with metastatic upper gastrointestinal (GI) cancers, such as pancreatic cancers, oesophago-gastric cancers and biliary tract cancers, does not exceed $10-11$ months, ${ }^{9-11}$ which is as poor as survival rates reported for patients with metastatic lung cancer. The standard of care for metastatic upper GI cancers is well described in the European Society of Medical Oncology guidelines. ${ }^{12-14}$ Briefly, the standard of care for metastatic pancreatic cancer in the first-line includes a combination of fluorouracil, irinotecan and oxaliplatin (FOLFIRINOX regimen) for patients without any cholestasis who are in good performance status; the standard of care includes gemcitabine monotherapy for frail patients. ${ }^{12}$ For metastatic biliary tract cancers, the standard of care includes a gemcitabine-based regimen (gemcitabine monotherapy, gemcitabine plus cisplatin or gemcitabine plus fluorouracil). ${ }^{13}$ Among patients with metastatic oesophago-gastric cancer, a few present with better prognosis and may be treated with a trastuzumab-based regimen, because they have been classified as HER2-positive tumours. ${ }^{14}$ For patients with metastatic HER2-negative tumours, various combinations of cytotoxics (fluoropyrimidines, taxanes, platinum compounds) may be offered to patients. ${ }^{12-14}$ Several experimental treatments (antiangiogenics, MET inhibitors, modulators of immune check points, new cytotoxics and so on) may be offered to these patients, but these treatments are restricted to patients in good health who are willing to participate in clinical trials; none of these treatments have produced a meaningful survival benefit thus far. In summary, patients with metastatic upper GI cancers do not benefit from currently available systemic therapies. Providing an extra survival benefit with EPC would be a considerable contribution for these patients, as would the implementation of these practices within the continuum of care of oncology in France.

\section{AIM OF THE STUDY}

We designed a randomised controlled trial, called EPIC, which aims to test the hypothesis that the use of EPC provides greater clinical benefits than standard practice for a population of patients with metastatic upper GI cancers. Overall survival (OS) will be used as a primary 
endpoint. The content of PC visits will be studied through a specific checklist. Patient-reported outcomes (quality of life, depression and anxiety) will also be investigated using dedicated and validated questionnaires.

\section{METHODS AND ANALYSIS \\ Study design}

This study was designed as a randomised, open-label, multicentre phase III trial. It aims to estimate the survival benefits of EPC combined with standard oncologic care (experimental arm) compared with standard oncology care only (standard arm) for patients with metastatic upper GI cancers (oesophago-gastric cancer, pancreatic cancer, biliary tract cancer). After the participant's eligibility is established, informed consent has been obtained and stratification factors are defined, the participant will be enrolled in the study and the treatment will be centrally allocated using the online ClinSight (CS) randomisation module from Clinsight software (Ennov, San Francisco, California, USA), ensuring the concealment of the next patient allocation. Treatments will be randomised in a 1:1 ratio, and a minimisation procedure will be used to balance patients according to centre, Eastern Cooperative Oncology Group (ECOG) performance status ${ }^{15}(0-1$ vs 2) and tumour location (oesophago-gastric, pancreas or biliary tract). Patients will be recruited nationwide from 17 university hospitals or cancer centres in France. Written informed consent will be obtained from the patient by an investigator before any screening or inclusion procedures. The patient will remain in the study until one of the following conditions applies: study withdrawal (patient or sponsor or investigator's decision) or death.

\section{Outcome measures}

\section{Study objectives}

The primary objective of this study is to evaluate the efficacy of EPC in terms of OS curves (intent-to-treat analysis). The secondary objectives are to assess the following: (1) the efficacy of EPC in terms of 1-year OS (intent-to-treat and per protocol analyses) and OS curves (per protocol analysis), (2) the patient-reported outcomes (quality of life, depression and anxiety) and the time until definitive deterioration (TUDD) for quality of life, (3) the number of patients receiving chemotherapy in their last 30 days of life, (4) the actual description of the PC package and (5) the presence or absence of advanced directives in patient files.

\section{Measurement tools}

OS is defined as the time between the date of randomisation and the date of death, no matter the cause of death. Patients who are alive at the cut-off date will be censored at that date. Quality of life will be assessed with the European Organisation for Research and Treatment of Cancer (EORTC) QLQ-C30 questionnaire. The QLQ-C30 aims to measure a person's overall quality of life, physical conditions and limits to the ability to carry out everyday activities; the questionnaire also assesses cognitive, emotional and social functioning as well as the appearance of symptoms frequently associated with cancer or its treatment. Patients are asked to check a scale that ranges from 1 to 4 (not at all, a little, quite a lot, a lot) or from 1 to 7 (from 1-very bad to 7-excellent). For each dimension, the QLQ-C30 score indicates definitive deterioration if the score decreases by more than 10 points compared with the score at baseline, without later improvement that is greater than 10 points compared with baseline unless the patient dropped out of the study, resulting in missing data. Thus, TUDD for the quality of life scores is defined as the time from randomisation to the first observation of a definitive deterioration of the QLQ-C30 score or the time from randomisation to death. Depression will be assessed with the HADS scale (Hospital Anxiety and Depression Scale). HADS aims to detect anxiety and depressive disorders. It contains 14 items with response options ranging from 0 to 3 : seven items assessing anxiety (score A) and seven items assessing depression (score D). The maximum score for a patient is 21 . The number of patients treated with chemotherapy in their last 30 days before death will also be recorded. PC visits will be performed by PC physicians. In both arms, all the dates of $\mathrm{PC}$ visits will be recorded in the database. The content of $\mathrm{PC}$ visits will be described through a specific checklist that will be completed by the PC physician after each visit. The number of patients in whom advanced directives are identified in medical records will be recorded.

\section{Patient selection criteria}

Inclusion criteria

Patients must:

- have an upper GI metastatic cancer, including pancreatic cancer, biliary tract cancer or gastric cancer (including junctional Siewert 2 and 3 cancers) (an amendment is being submitted to our ethic committee in order to include other oesophageal cancers, too),

- be 18 years of age or older,

- have an ECOG performance status $\leq 2$,

- be planned for treatment with first-line Ct,

- have a life expectancy of more than 4 weeks,

- have a good understanding of the French language,

- have health insurance coverage,

- sign and date a written informed consent form.

\section{Exclusion criteria}

Patients with any of the following conditions or characteristics are excluded from the study:

- locally advanced cancer,

- junctional Siewert one oesophago-gastric cancer (An amendment is being submitted to our ethic committee in order to include these cancers together with other oesophageal cancers),

- gastric or junctional oesophago-gastric cancer with dysphagia,

- gastric or junctional oesophago-gastric cancer with unknown or positive HER2 status, 


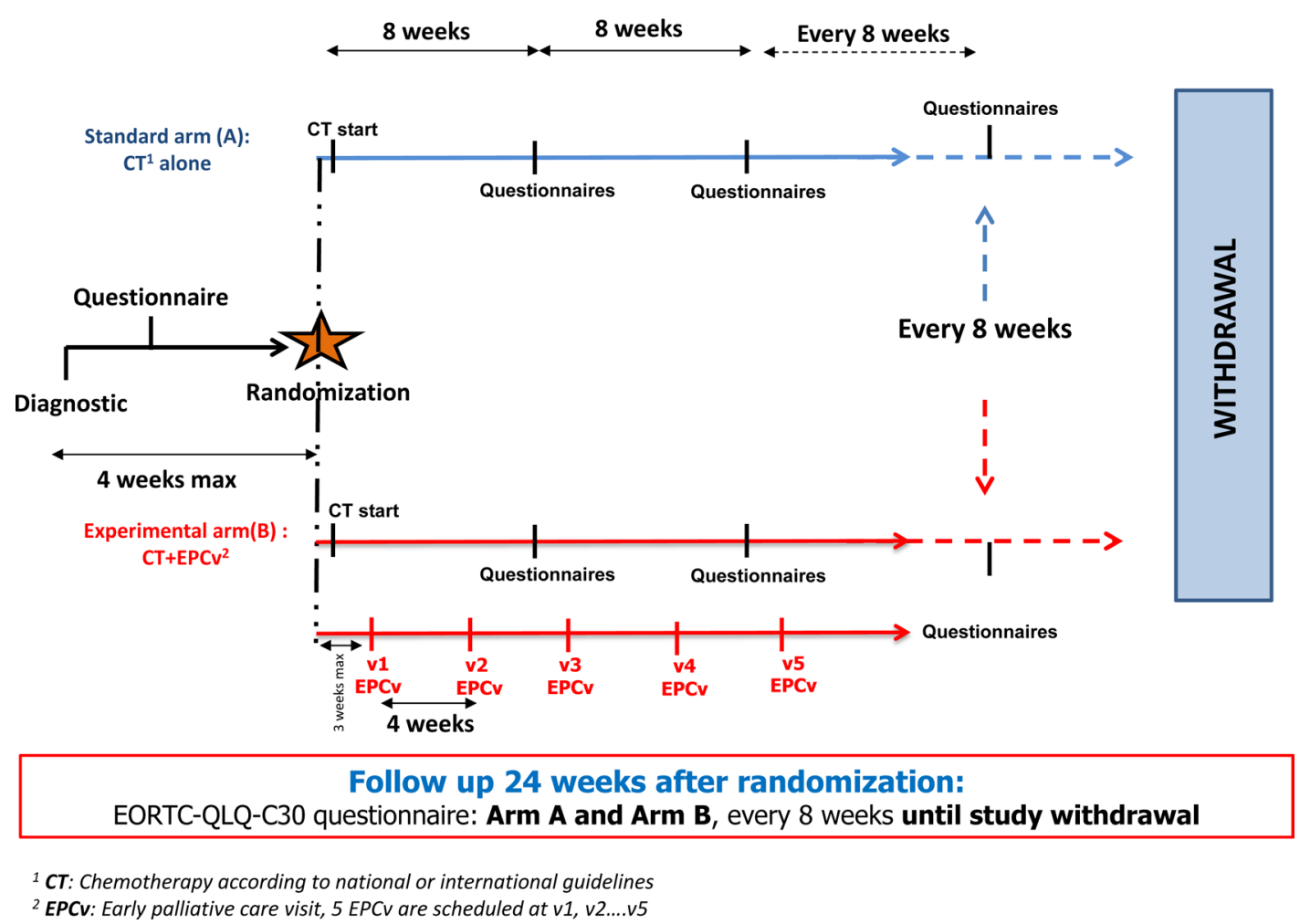

Figure 1 Study design.

- compression of the biliary tract without any bypass procedure.

\section{STUDY DESCRIPTION Intervention}

Medical oncologists will be in charge of the patient for Ct administration and for supportive care, in accordance with professional practices. PC specialists will be in charge of PC/EPC visits. In order to match with standard practice in France, participants allocated to the standard arm (Ct alone) are not scheduled to meet with the PC service, but a PC visit can be performed anytime if requested by the patient, the family or the oncologist. In the experimental arm $(\mathrm{Ct}+\mathrm{EPC})$, five PC visits are scheduled (figure 1). The first visit (V1) will be scheduled within the first 3 weeks after randomisation. The remaining four visits will be scheduled every month. The content of each of the five PC visits will be described by the PC physician and documented in the database following a specific checklist developed by PC physicians. In part, the visits will focus on the following items:

- Discussion with the patient, focusing on his/her understanding of the disease, its treatment and the PC process.

- Evaluation of clinical status and symptoms.

- Evaluation of psychological status.

- Evaluation of the social environment, including the patient's way of living.

- Stakeholder needs: psychologist, physiotherapist, dietician, social worker, and so on.

- Caring for the patient and his/her family.
- Discussion about the identification of the "person of trust' and about advanced directives.

- Coordination and continuum of care.

The choice of first-line $\mathrm{Ct}$ will be decided by each investigator but should adhere to national or international guidelines. If CT is stopped for any reason (toxicity, disease progression or deterioration of health status), the patient will remain in the study.

\section{Data collection}

At baseline, before randomisation, patients will have to complete the EORTC-QLQ-C30 and the HADS questionnaires. During the study, the EORTC-QLQ-C30 and the HADS questionnaires will be completed by patients every 8 weeks after randomisation. Then, 24 weeks after randomisation, only the EORTC-QLQ-C30 questionnaire will be completed by patients every 8 weeks until the end of the study. In both arms, the number and the dates of PC/EPC visits that are performed will be recorded. The number of patients in whom advanced directives are identified in medical records will also be recorded.

\section{STATISTICAL CONSIDERATIONS}

To ensure an $80 \%$ power, 381 deaths are required to show a significant difference in OS curves if there is an absolute difference of $10 \%$ in 1-year OS rates $(40 \%$ vs $50.3 \%$, $\mathrm{HR}=0.75$; log rank test two-sided alpha $=5 \%$ ), assuming proportional hazards over time. Assuming an exponential distribution of survival time, with an accrual duration of 3 years, a 1-year minimum follow-up and a final analysis 
at 4 years, it will be necessary to randomise 480 patients (240 in each group). This calculation takes into account a yearly $2 \%$ loss to follow-up rate. An efficacy interim analysis is planned for when approximately 190 deaths are observed (which is expected to occur 27 months from the start of the study). The significance level is fixed at $p=0.003$ for the interim analysis and $p=0.049$ at the final analysis (Lan-DeMets alpha-spending function) ${ }^{16}$ with an O'Brien-Fleming efficacy boundary ${ }^{17}$ No futility analysis is planned as the proportional hazards assumption may not be met; there may be a larger treatment effect with a longer follow-up period than in the first part of the survival curves. The interim analysis will also evaluate whether the sample size of the EPIC trial should be increased, considering the observed OS curve in the control group.

OS curves will be estimated using the Kaplan-Meier method. After checking the proportional hazards assumption, the treatment effect of the experimental arm compared with the control arm, in terms of OS, will be based on the estimation of the HR of death in a Cox model (HR-death, based on the comparison of the OS curves between the two treatment groups) and tested against the null hypothesis of no treatment effect using a log rank test with a two-sided alpha of $5 \%$. The proportional hazards assumption underlying the HR estimate in Cox models will be evaluated using graphic methods and models, including interaction with time. Appropriate methods for estimating treatment effect will be used if the proportional hazard assumption appears to be violated or questionable (use of the restricted mean survival as published by Royston and Parmar). ${ }^{18}$ Heterogeneity of the treatment effect by stratification factors will be evaluated using forest plots and interaction tests. The main analyses will be performed on the intent-to-treat dataset, including data from all patients in the treatment group allocated by randomisation until their last follow-up visit. A sensitivity analysis is also planned on the per protocol dataset in which patients in the standard arm who completed more than one PC visit within the first 6 months of treatment after randomisation will be censored at the date of their second $\mathrm{PC}$ visit, and patients in the treatment arm who completed fewer than five EPC visits within the first 6 months after randomisation will be censored at the date of the first missing EPC visit. One-year survival rates with their 95\% CI will also be estimated and compared between groups, considering the intent-to-treat and the per protocol datasets.

Quality of life will be analysed according to the EORTC manual recommendations. For each dimension, patients with at least one score will be included in the analysis. Patients without a follow-up QLQ-C30 score will be censored just after baseline. Patients without baseline scores will be censored at baseline. TUDD curves for both arms will be calculated using the Kaplan-Meier method and described using medians and $95 \%$ CIs.

An independent data monitoring committee will meet when the results of the planned interim analysis are available (ie, when 190 patients have died) to review the results of the first efficacy interim analysis and to re-estimate the sample size if the baseline OS rate differs from the protocol assumptions.

\section{ETHICS AND DISSEMINATION \\ Ethical considerations}

This clinical trial will be conducted in accordance with the Declaration of Helsinki ${ }^{19}$ or the laws and regulations of the country, whichever provides greater protection to the patient. This study follows the International Conference on Harmonisation E6 Guideline for Good Clinical Practice, reference number CPMP/ICH/135/95. ${ }^{20}$ The protocol has been examined by the Patient Committee of the National League against Cancer, paying particular attention to the quality of the information letter, to the monitoring plan and to suggestions implemented into the protocol to improve the comfort of the patients. An independent data monitoring committee for the trial will be formed to guarantee protection of the patients, to ensure that the trial is conducted in an ethical fashion and to evaluate the risk/benefit ratio of the trial by reviewing the interim results of the trial. The study protocol has been approved by our local ethics committee (CPP Nord-Ouest I, 4 April 2016).

\section{DISSEMINATION}

The study is registered at clinicaltrials.gov (NCT02853474). The protocol and the trial results, even if they are inconclusive, will be presented at international oncology congresses and published in peer-reviewed journals.

\section{DISCUSSION}

This EPIC trial was set up in September 2016. It is a randomised trial primarily designed to detect an OS benefit due to EPC combined with standard oncologic care compared with standard oncologic care only for patients with metastatic upper GI cancer. The design of EPIC differs from the design of the seminal trials by Temel and colleagues, ${ }^{5}$ which demonstrated that EPC improves quality of life (the primary objective of their trial) and may improve OS (a secondary objective) for patients with advanced cancers.

One may argue that the main motivation for many oncologists to engage with EPC is to enhance quality of life for their patients throughout the cancer journey. This is precisely what Temel et al did. ${ }^{5}$ When using OS as the primary endpoint of EPIC, as we will, there is a theoretical danger that if a study does not meet its OS endpoint, it will indicate that EPC has 'failed' and should be discarded. Our point is different. Our country has a strong culture of integrating PC into oncology services. However, despite efforts from many PC professionals, PC is frequently offered to patients at a late stage of their metastatic disease. Some components of PC visits, such as visits with a dietician and/or with psychologists, are 
usually offered at an earlier stage but not as systematically as they should be. Using OS as the primary endpoint of EPIC, we postulate that without a strong 'signal', such as a survival benefit, sent to medical oncologists and colleagues in charge of metastatic patients with upper GI malignancies, it would take some time before the concept of EPC is implemented in our country. Furthermore, the benefits of EPC have yet to be validated in a population of patients with metastatic upper GI cancers. Patients with metastatic upper GI malignancies are different from patients with metastatic lung cancers; they do not present the same, and we assume that their comorbidities and their treatment-related symptoms are also different. The difference in terms of reduced risk of death $(-25 \%)$ that we have chosen for the primary outcome is derived from the work reported by Temel et al. $(-40 \%)$ regarding metastatic lung cancers. ${ }^{5}$ Further reducing the risk of death to $25 \%$ should lower the theoretical danger that this study may not meet its OS endpoint.

In Temel's trial, ${ }^{5}$ the content of the EPC package, which was rather vague, was adapted from American guidelines for PC visits. ${ }^{6}$ There are no such recommendations in our national context. To overcome this, PC specialists have developed a checklist of all of the items that could be addressed within PC coverage. Hence, one of the secondary endpoints of this EPIC trial will be to make an actual description of each EPC/PC visit as well as to provide a description of the whole EPC/PC package. At the end of the study, the materials we will collect should help us in drafting guidelines for PC in France.

To conclude, we expect that this study will lead to earlier integration of PC in oncologic care for patients with metastatic GI cancer.

Acknowledgements The authors wish to warmly thank Séverine Marchant for editing this manuscript.

Contributors $A A, A D S$, EH and M-CLD designed the study and will contribute to data interpretation. AA, ADS, M-CLD, EH and SLL-D contributed to the drafting of the manuscript. AA, EH and SLL-D contributed to the trial set-up. SLL-D is responsible for data collection and for administrative support. EB will contribute to statistical analysis. M-CLD is responsible for data management and statistical analysis. All authors contributed to the revision of the manuscript and approved it for submission.

Funding This study is supported by unrestricted public grants from Conseil Régional du Nord Pas-de-Calais and from caregivers Ligue National contre le Cancer. The funders have no role in study design, management, analysis and interpretation of data as well as no role in the writing of the final report.

Competing interests None declared.

Patient consent Obtained.

Ethics approval (CPP Nord-Ouest I, 4 April 2016).

Provenance and peer review Not commissioned; externally peer reviewed.

Data sharing statement This manuscript contains original material without any unpublished data, but the full results of this ongoing trial.
Open Access This is an Open Access article distributed in accordance with the Creative Commons Attribution Non Commercial (CC BY-NC 4.0) license, which permits others to distribute, remix, adapt, build upon this work non-commercially, and license their derivative works on different terms, provided the original work is properly cited and the use is non-commercial. See: http://creativecommons.org/ licenses/by-nc/4.0/

(c) Article author(s) (or their employer(s) unless otherwise stated in the text of the article) 2018. All rights reserved. No commercial use is permitted unless otherwise expressly granted.

\section{REFERENCES}

1. SFAP. http://www.sfap.org/content/definition-des-soins-palliatifs-etde-laccompagnement. accessed 6 Jan 2017

2. Bakitas MA, Tosteson TD, Li Z, et al. Early versus delayed initiation of concurrent palliative oncology care: patient outcomes in the ENABLE III randomized controlled trial. J Clin Oncol 2015;33:1438-45.

3. http://www.who.int/cancer/palliative/fr. accessed 6 Jan 2017

4. Loi $n^{\circ}$ 99-447 Du 9 juin 1999 visant à garantir le droit à l'accès aux soins palliatifs. JORF $n^{\circ} 132$ du 10 juin 1999.

5. Temel JS, Greer JA, Muzikansky A, et al. Early palliative care for patients with metastatic non-small-cell lung cancer. $N$ Engl J Med 2010;363:733-42.

6. Clinical practice guidelines for quality palliative care. 2 nd ed. Pittsburgh: National Consensus Project, 2009. www.nationalconse nsusproject.org. (accessed 23Jul 2010).

7. Pirl WF, Greer JA, Traeger L, et al. Depression and survival in metastatic non-small-cell lung cancer: effects of early palliative care. $J$ Clin Oncol 2012;30:1310-5.

8. Schnipper LE, Smith TJ, Raghavan D, et al. American Society of clinical oncology identifies five key opportunities to improve care and reduce costs: the top five list for oncology. J Clin Oncol 2012;30:1715-24.

9. Conroy T, Desseigne F, Ychou M, et al. FOLFIRINOX improves survival in patients with metastatic pancreatic Cancer. N Engl J Med 2011;364:1817-25.

10. Lordick F, Kang YK, Chung HC, et al. Capecitabine and cisplatin with or without cetuximab for patients with previously untreated advanced gastric cancer (EXPAND): a randomised, open-label phase 3 trial. Lancet Oncol 2013;14:490-9.

11. Malka D, Cervera P, Foulon S, et al. Gemcitabine and oxaliplatin with or without cetuximab in advanced biliary-tract cancer (BINGO): a randomised, open-label, non-comparative phase 2 trial. Lancet Oncol 2014;15:819-28.

12. Ducreux M, Sa Cuhna A, Caramella C, et al. Cancer of the pancreas: esmo Clinical Practice guidelines. Ann Oncol 2015;26(suppl 5):v56-v68.

13. Valle JW, Borbath I, Khan SA, et al. Biliary cancer: esmo clinical practice guidelines for diagnosis, treatment and follow-up. Ann Oncol 2016;27(suppl 5):v28-v37.

14. Smyth EC, Verheij M, Allum W, et al. Gastric Cancer: esmo clinical Practice guidelines. Ann Oncol 2016;27(suppl 5):v38-v49.

15. Oken MM, Creech $\mathrm{RH}$, Tormey DC, et al. Toxicity and response criteria of the Eastern Cooperative Oncology Group. Am J Clin Oncol 1982;5:649-56.

16. Lan KKG, DeMets DL. Discrete Sequential Boundaries for clinical trials. Biometrika 1983;70:659-63.

17. O'Brien PC, Fleming TR. A multiple testing procedure for clinical trials. Biometrics 1979;35:549-56.

18. Royston P, Parmar MK. The use of restricted mean survival time to estimate the treatment effect in randomized clinical trials when the proportional hazards assumption is in doubt. Stat Med 2011;30:2409-21.

19. World medical association. http://www.wma.net/en/30publications/ 10policies/b3/17c.pdf. (accessed 6 Jan 2017).

20. ICH. http://www.ich.org/products/guidelines/efficacy/efficacy-single/ article/general-considerations-for-clinical-trials.html. (accessed 6 Jan 2017). 
Correction: Impact of early palliative care on overall survival of patients with metastatic upper gastrointestinal cancers treated with first-line chemotherapy: a randomised phase III trial

Hutt E, Da Silva A, Bogart E, et al. Impact of early palliative care on overall survival of patients with metastatic upper gastrointestinal cancers treated with first-line chemotherapy: a randomised phase III trial. BMJ Open 2018;8:e015904. doi: 10.1136/ bmjopen-2017-015904

This article was previously published with an error.

The funding information in the published article was incomplete. The complete funding details are as follows:

This study is supported by unrestricted public grants from Conseil Régional du Nord Pas-de-Calais and from caregivers Ligue National contre le Cancer. The EPIC trial (NCT02853474.) is also supported by public grants from the French National Cancer Institute, INCa (INCa-DGOS_11170). The funders have no role in study design, management, analysis and interpretation of data as well as no role in the writing of the final report.

These errors did not affect the design or results of the study.

Open access This is an open access article distributed in accordance with the Creative Commons Attribution Non Commercial (CC BY-NC 4.0) license, which permits others to distribute, remix, adapt, build upon this work non-commercially, and license their derivative works on different terms, provided the original work is properly cited, appropriate credit is given, any changes made indicated, and the use is non-commercial. See: http://creativecommons.org/licenses/by-nc/4.0/.

(C) Author(s) (or their employer(s)) 2019. Re-use permitted under CC BY-NC. No commercial re-use. See rights and permissions. Published by BMJ.

BMJ Open 2019;9:e015904corr1. doi:10.1136/bmjopen-2017-015904corr1

A) Check for updates 